\title{
Studies of radiation fields of LCLS-II super conducting radio frequency cavities
}

\author{
M. Santana Leitner \\ Radiation Protection Department, \\ SLAC National Accelerator Laboratory, 2575 Sand Hill Road, \\ Menlo Park, CA 94025, USA \\ msantana@slac.stanford.edu \\ L. Ge, Z. Li, C. Xu, C. Adolphsen, M. Ross and M. Carrasco \\ SLAC National Accelerator Laboratory, 2575 Sand Hill Road, \\ Menlo Park, CA 94025, USA
}

Published 1 September 2016

\begin{abstract}
The Linac Coherent Light Source II (LCLS-II) will be a hard X-ray Free Electron Laser whose linac can deliver a 1.2 MW CW electron beam with bunch rates up to $1 \mathrm{MHz}$. To efficiently generate such a high power beam, Super-Conducting Radio-Frequency (SCRF) cavities will be installed in the upstream portion of the existing $3 \mathrm{~km}$ Linac at the SLAC National Accelerator Laboratory. The 9-cell niobium cavities will be cooled at $2 \mathrm{~K}$ inside 35 cryomodules, each containing a string of eight of those cavities followed by a quadrupole. The strong electromagnetic fields in the SCRF cavities will extract electrons from the cavity walls that may be accelerated. Most such dark current will be deposited locally, although some electrons may reach several neighboring cryomodules, gaining substantial energy before they hit a collimator or other aperture. The power deposited by the field emitted electrons and the associated showers may pose radiation and machine protection issues at the cryomodules and also in other areas of the accelerator. Simulation of these effects is therefore crucial for the design of the machine. The in-house code Track3P was used to simulate field emitted electrons from the LCLS-II cavities, and a sophisticated 3D model of the cryomodules including all cavities was written to transport radiation with the Fluka Monte Carlo code, which was linked to Track3P through custom-made routines. This setup was used to compute power deposition in components, prompt and residual radiation fields, and radioisotope inventories.
\end{abstract}

Keywords: LCLS-II; Field Emission; SCRF; Cryomodule; Radiation; Track3P; FLUKA; ILC.

\section{LCLS-II}

Since 2009, the Linac Coherent Light Source (LCLS) hard X-ray Free Electron Laser has delivered electron pulses at $120 \mathrm{~Hz}$ by using the last third of the SLAC 2-mile linac, followed by a transfer line and up to 30 undulators, where the coherent radiation is generated. LCLS-II will add a 4.0 GeV, $1 \mathrm{MHz}$, CW SCRF electron accelerator in the first 700 meters of the Linac, as well as additional transport beam lines and new,

This is an Open Access article published by World Scientific Publishing Company. It is distributed under the terms of the Creative Commons Attribution 3.0 (CC-BY) License. Further distribution of this work is permitted, provided the original work is properly cited. 
adjustable gap polarized undulators, to produce tunable, fully coherent X-rays in programmable bunch patterns.

LCLS-II's high beam power (1.2 MW) is generated in 35 cryomodules, each with eight $1.3 \mathrm{GHz}$ SCRF cavities operating at $\sim 16 \mathrm{MV} / \mathrm{m}$. The cavities are made of nine niobium cells cooled by liquid helium at $2 \mathrm{~K}$. The cryomodules are grouped in four linac segments (L0 to L3), with one cryomodule in L0 (750 keV $\rightarrow 100 \mathrm{MeV})$, two in L1 (100 $\mathrm{MeV} \rightarrow 250 \mathrm{MeV}), 12$ in L2 $(250 \mathrm{MeV} \rightarrow 1.6 \mathrm{GeV})$ and 20 in $\mathrm{L} 3(1.6 \mathrm{GeV} \rightarrow 4.0 \mathrm{GeV})$.

Cavity apertures are intentionally large $(70 \mathrm{~mm})$ so that dark current from the gun and beam halo from gas bremsstrahlung and Touschek do not irradiate the cavities, and instead are mostly filtered out in the bunch compression and collimator sections $(\mathrm{LH}$, BC1-3) located between the linac segments. However, dark current will also be emitted from the cavity walls due to the strong RF fields, some of which will be accelerated along the linac (i.e. captured). Because the dark current energy is generally low compared to the beam, much of it will be swept out of the beam line by the quadrupole magnets at the end of each cryomodule, thereby irradiating the cryomodules and the tunnel.

\section{Scheme to Compute Radiation Fields at LCLS-II Cryomodules}

Analytical formulae, RF solvers and Monte Carlo codes have been traditionally used ${ }^{1,2}$ to address various questions involving dark current from cavities. Generally only a few cavities are simulated and geometries are simplified, or the calculations focus only on beam dynamics. Here we expand the scope of a 2003 TESLA study, ${ }^{3}$ simulating the surface emission of electrons within complex cavity shapes, their trajectory and energy variation in 3D electromagnetic fields, their transport between cryomodules including the effect of quadrupoles at different gradient settings, the subsequent re-acceleration in up to eight contiguous cryomodules, and ultimately the showers of lost electrons through a detailed model of the beam line, including damage estimates to components, inventories of activation products, and residual dose rates.

To cope with such a task, state-of-the-art codes in the domains of cavity design (Track3P) and radiation transport (Fluka) have been linked through extensive coding, and detailed models of the fields and geometry have been implemented for both of them.

\subsection{Track3P}

Track $3 \mathrm{P}^{4}$ is a $3 \mathrm{D}$ finite element particle tracking code with a grid of curved elements that fits the curvature of cavity boundaries, allowing high-fidelity modeling of the geometry and correct emission angles for particles. The parallel implementation enables handling large problems with reasonable simulation times.

Primary emission of electrons from RF fields is modelled according to the standard Fowler-Nordheim ${ }^{5}$ formula of Eq. (1), where the emission current density $\left(J\right.$ in $\left.\mathrm{A} / \mathrm{m}^{2}\right)$ as a function of position and time $(r, t)$ is determined by the work function of the material $(\varphi$ in $\mathrm{eV})$ and the product of the strength of the surface electric field $(E \mathrm{in} \mathrm{V} / \mathrm{m})$ and the local field enhancement factor $(\beta)$ : 


$$
J(r, t)=1.54 \cdot 10^{\left(-6+\frac{4.52}{\sqrt{\varphi}}\right)} \cdot \frac{(\beta \cdot E(r, t))^{2}}{\varphi} \cdot e^{-\left(\frac{6.53 \cdot 10^{9} \cdot \varphi^{1.5}}{\beta \cdot E(r, t)}\right)} .
$$

In this study, primary field emitted particles were generated within one quarter of the cross section whenever the electric field exceeded the emission threshold, and tracking was performed for up to $60 \mathrm{RF}$ cycles. Randomization over the $2 \pi$ angle was carried out during post-processing.

The dark current module in Track3P simulates the evolution of the field emitted electrons and calculates the dark current capture and the surface heating due to the bombardment of the dark current electrons on the cavity wall. The RF fields for the particle tracking are imported from SLAC's finite element field solvers. ${ }^{6}$

Monitors were implemented to record dark current impacts on cavity walls as well as escaping current through the ends of the 8-cavity strings of the cryomodules.

\subsection{Fluka}

Fluka $^{7,8}$ is a general purpose radiation transport code that uses microscopic models to simulate interactions with matter and with magnetic fields of about 60 different particles (and also ions) in an energy range generally spanning between $1 \mathrm{keV}$ and $10000 \mathrm{TeV}$. Numerous subroutines are user configurable, allowing for extensive customization.

Among other custom physics options, photonuclear and photo-muon reactions were included (and statistically enhanced). For activation studies, heavy fragment evaporation and fragment coalescence were also switched on, and transport thresholds for prompt radiation were raised.

A prototype of a cavity was implemented in Fluka geometry by fitting the surface profile of each of the nine bulb-shaped cells with eleven quadrics (cylinders, ellipsoids, cones and paraboloids), and then adding other components like the helium cooling tank, flanges, etc. The resulting geometry was overlaid on engineering drawings to ensure minimal discrepancies, and instructions were coded in Fluka source subroutine to recurrently apply micro adjustments to Track3P impact coordinates so those would be within a small tolerance to the cavity inner surface.

Using Fluka lattice capabilities, the cavity prototype was replicated along the beam line according to the positions defined in the accelerator deck files. In a similar manner, a single cryomodule prototype was coded and copied eight times for a total of nine cryomodules. Besides cavity tanks, the cryomodule geometry includes the outer shell, all layers of magnetic and thermal insulation, and all cooling supply and return lines. A Flair ${ }^{9} 3 \mathrm{D}$ rendering of the Fluka geometry and a zoom-in of one cavity are shown in Fig. 1. Fluka does not yet offer the possibility to include the effect of electric fields on charged particles. Since this effect is fundamental for this study, acceleration of field emitted electrons was entirely pre-calculated with Track3P. 


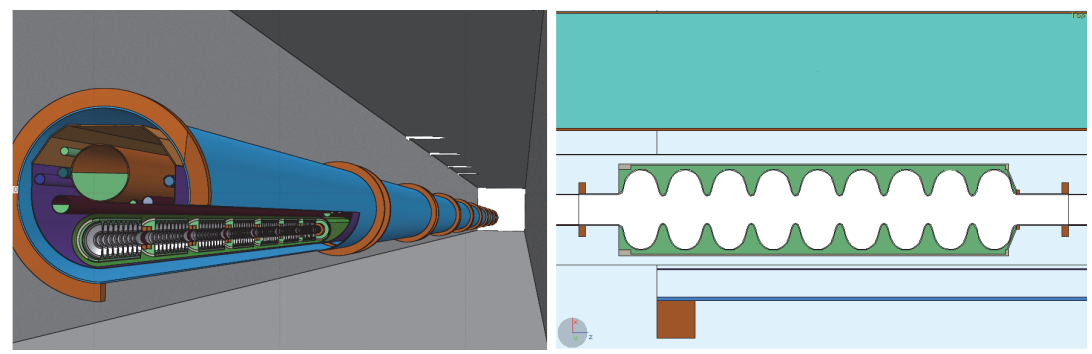

Fig. 1. The current Fluka model of LCLS-II has nine cryomodules, each with eight 9-cell cavities, a quadrupole, cooling vessels, supply/return lines, heat/magnetic shields, etc.

\section{3. 'Soft' coupling of Track3P and Fluka}

For an exact treatment of this problem, Track3P should initiate the simulation and call Fluka whenever interaction with matter occurs, and Fluka should in turn call Track3P when a new charged particle entered a region with RF field. Such a full coupling scheme would require access to and modification of both source codes and might run too slowly to achieve statistically significant results. Alternatively, a two-phase scheme was designed that allowed executing Track3P and Fluka in series.

In the first phase, Track3P computes field emission and tracks electrons up to the collision/escape boundaries. For a single powered cryomodule, this is trivial, as Track3P has a full model of the cryomodule. However, if there is more than one cryomodule (like in most applications), then Fluka takes the particles escaping from one cryomodule computed by Track3P and performs the transport through the quadrupole and space between cryomodules. It records the coordinates (position, momentum, time, etc.) of the particles as they enter the next/previous cryomodule (the cryomodules are spaced such that electrons are accelerated downstream and upstream equally). The z-coordinate is adjusted to the start/end position of the single cryomodule Track3P model, so that Track3P can compute RF transport of those particles using its standard LCLS-II 8-cavity model. This process is repeated up to eight times, meaning that particles born in a given cryomodule (CM 0) are tracked as they collide or get accelerated in neighboring cryomodules $\mathrm{CM} \pm 1$ through $\mathrm{CM} \pm 8$. In total, up to17 impact collision files are created in this phase (step0.dat, ... step8.dat, stem1.dat ... stem8.dat).

In the second phase, radiation transport of impact electron data is performed for the Fluka model, which includes nine back-to-back cryomodules. For each history, Fluka will start by randomizing the cryomodule reference start position. Next it will randomly select an event from all impact files, and it will determine the starting absolute $\mathrm{z}$ coordinate as a function of the reference cryomodule and the step number corresponding to the impact file. Then tracking of electrons and subsequent showers though matter and magnet fields is performed.

To achieve sensible results, the following two considerations had to be programmed through subroutines, using particle latching and transferring common variable arrays: 
(i) Primary electrons (not those from showers) must be terminated if they enter a cryomodule other than the one where they started to be tracked in phase 2 . This is required to avoid double counting (such events are considered in higher order impact data) and also because Fluka cannot simulate RF acceleration.

(ii) Only events from step0.dat impact file contribute to the total weight by which events are normalized (see next section), as histories of higher order files just represent track portions of events from step 0 .

\subsection{Normalization of results; extracted dark current.}

As mentioned just above, raw results are expressed in terms of the sum of statistical weights (Weipri) of all primary field emitted electrons that have contributed to the results. Analogous to simulations involving the accelerator beam, in which results are expressed in terms of beam power (e.g. Gy/W), the results here will be scaled to the dark current escaping a string of eight cavities (also called the captured current), for which measurements exist from experimental set-ups at different gradient settings.

To perform such renormalization, it is necessary to know the fraction of dark current that escapes the cryomodules. For that sake, detectors were added to Fluka simulations to count the charge $(Q)$ of field emitted electrons escaping their birth cryomodule as a function of the total field emission (Weipri). By multiplying $Q$ by the RF frequency $(v=$ $1.3 \times 10^{9} \mathrm{~Hz}$ ), a normalized field emission extraction current of $I_{ \pm}=7.9 \times 10^{-4} \mathrm{nA} /$ Weipri is obtained. This number is independent of the quadrupole gradient and nearly independent of the beam direction. Thus, Fluka estimators $\zeta$ (unit/Weipri) must be scaled as Eq. (2):

$$
\frac{\langle\zeta\rangle}{\langle Q\rangle}=\frac{\zeta\left[\frac{\text { unit }}{\text { Weipri }}\right]}{Q\left[\frac{C}{\text { Weipri }}\right]} \cdot \frac{v}{v}=\frac{1.3 \cdot 10^{9} \cdot \zeta\left[\frac{\text { unit }}{\text { Weipri }}\right]}{I_{ \pm}\left[\frac{n A}{\text { Weipri }}\right]}=1.64 \cdot 10^{12} \cdot \zeta\left[\frac{\text { unit }}{s \cdot n A}\right] .
$$

Although experimental values of captured current $\left(I_{c}\right)$ are notoriously scattered, $I_{c}=$ $1-10 \mathrm{nA} /$ cryomodule is a reasonable upper-end operational range for LCLS-II. ${ }^{10}$ For safety applications, such as build-up of residual dose, $I_{c}=10 \mathrm{nA}$ will be assumed, while for shielding purposes of prompt dose, excursions to $1 \mu \mathrm{A}$ should also be considered.

\section{Results}

Simulations were run for three different quadrupole gradients. In the first case, labeled ' 0 $\mathrm{MeV}$ ', the quads are off, which represents the lattice strength at L0, as well as the case where the quads are inadvertently shut off. The second gradient, $3.17 \times 10^{-3} \mathrm{~T} / \mathrm{cm}$, corresponds to $700 \mathrm{MeV}$ beams and represents the L2 region. The last setting, $2.48 \times 10^{-2}$ $\mathrm{T} / \mathrm{cm}$, is the value at the highest beam energy, $4 \mathrm{GeV}$ (downstream end of L3).

\subsection{Field emission balance and forward/backward transmission}

Only $3.5 \%$ of the total dark current charge escapes at either end of the 8-cavity string. However, those electrons carry as much as $80 \%$ of the total $116 \mathrm{~mW} / \mathrm{nA}$ power $(40 \%$ in each direction). Thus, independent of the quadrupole settings, each LCLS-II cryomodule 
will absorb about $20 \%$ of its generated dark current power, i.e. $23 \mathrm{~mW} / \mathrm{nA}$. Additionally, the escaping field emission, which can gain further energy in the neighboring cryomodules, will be swept out of the beam line by the quadrupoles (with the fraction depending on the quad strength), thus leading to power loss in other cryomodules.

\subsubsection{Power of multiply-accelerated dark current}

If quadrupoles are set at high field strengths, e.g. for $4 \mathrm{GeV}$ primary beams, then large amounts of dark current will be lost at each transition between cryomodules, and the transmission of dark current along the beam line will decay rapidly. However, if the quadrupoles are not powered, then adiabatic damping during acceleration will limit the beam losses, and the beam power will increase. At some intermediate quadrupole setting (here $\sim 700 \mathrm{MeV}$ ), dark current power transmission is roughly constant due to the balance between transmission losses between cryomodules and the energy gains at cavities. For these cases, Fig. 2 (Left) illustrates the beam power propagation eight cryomodules upstream and downstream from the one generating the current, as described in Sec. 2.3.
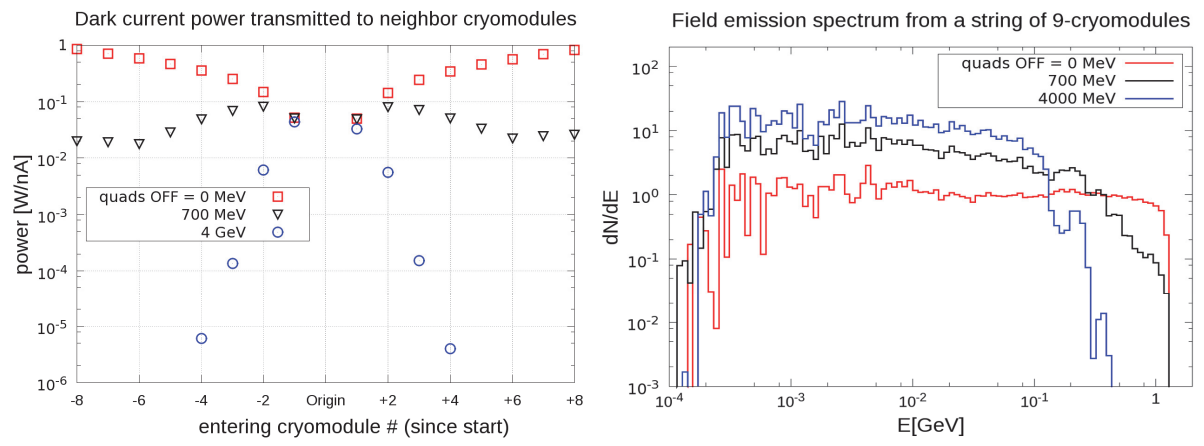

Fig. 2. (Left): Field emission power [W/nA] transmitted from the source cryomodule to the \pm 8 neighboring cryomodules. (Right): the energy spectra $[\mathrm{dN} / \mathrm{dE}]$ of the field emitted beam at either end of the string.

For several radiation and machine protection studies, it is necessary to have an estimate of the maximum cavity dark current power $(M P)$ at either end of a linac segment. The longest segment is L3 with 20 cryomodules, and as seen above, the maximum power transmission occurs with the quadrupoles off. Thus, fitting a curve to the red squares of Fig. 1 (left) and integrating for 20 cryomodules, a maximum power of $25.3 \mathrm{~W} / \mathrm{nA}$ could potentially impact both ends of L3, per Eq. (3) below:

$$
M P_{20}(k=0)=0.056 \cdot \sum_{N=1}^{20} N^{1.3}=25.3\left[\frac{W}{n A}\right] .
$$

As stated previously, we expect $I_{c}$ to be less than $10 \mathrm{nA}$, but in exceptional cases it could reach the $\mu \mathrm{A}$ level. Therefore, collimation sections BC2 and BC3 (at either end of L3) must be able to absorb $\sim 25 \mathrm{~kW}$ from L3. Likewise, the 12 cryomodules of L2 could induce dark current powers of $8.1 \mathrm{~kW} / \mu \mathrm{A}$ in $\mathrm{BC} 1$ and $\mathrm{BC} 2$ with the quads off. 


\subsubsection{Spectrum of multiply-accelerated dark current}

Figure 2 (right) shows the energy spectra of the above-described dark current beams at each end of a cryomodule string for the three quadrupole gradients. By extrapolating the red curve, corresponding to quads-off, to the L2 and L3 lengths, the resulting spectra would be roughly flat in log-log coordinates, ranging from thermal energy to $1.44 \mathrm{GeV}$ to $2.40 \mathrm{GeV}$, respectively.

\subsubsection{Radiation decay as a function of distance from single cavity test}

During machine commissioning, cavities will be tested individually with all other cavities powered off. Because other components would ideally need to be tested and installed during that time, it is necessary to understand what the radiation levels would be in the tunnel in view of authorizing access to some areas.

For this study, no field-emission acceleration in neighboring cryomodules was required, as those will not be powered. Thus, Fluka source card was set to read only a primary impact file (step0.dat) for a single powered cavity. To be conservative, all quadrupoles were assumed unpowered ( $0 \mathrm{MeV}$ gradient).

At the powered cavity, results showed unacceptably high dose equivalent rate levels even in the aisle $(50 \mathrm{mSv} / \mathrm{h} / \mathrm{nA})$, while at $50 \mathrm{~m}$ distance (up or downstream) similar values would be found at the beam pipe radius where installations may be taking place. Also, field-emission might be higher during commissioning, ${ }^{11}$ and the corresponding detection system may not yet be installed or commissioned at that point. Thus, it has been decided that no access should be granted to the linac during commissioning of the cold cryomodules, even for individual cavities.

\subsection{Lateral radiation}

Section 3.1.1 showed that for low quadrupole fields, the dark current power is mostly deposited at the warm sections between the linac segments. Otherwise, most dark current power is deflected by the quadrupoles, and cryomodules receive most of the radiation load. This can lead to potential damage of electromechanical components, sensors and wire insulation inside the cryomodules, as well as damage or malfunction of electronics and instrumentation in the tunnel. Predictions of radiation levels near the cryomodules are thus necessary to define what materials should be used, where to place electronic racks, if any, as well as to plan whether service of hard-to-access components will be required.

Figure 3 shows elevation fluence maps for a 9-cryomodule string at the three quadrupole settings considered above. The middle plot, obtained for the $700 \mathrm{MeV}$ quadrupole setting, displays the highest radiation fields as the dark currents are only partially swept out at each quad, allowing the transmitted portion to gain energy at nearly the same rate that the current diminishes. Thus, most radiation damage and activation issues are expected to occur in the L2 section, followed by L3. The following studies 
have been performed for the L2 case, with focus on the central cryomodule in the model (CM 0) where the contribution from both sides is significant.

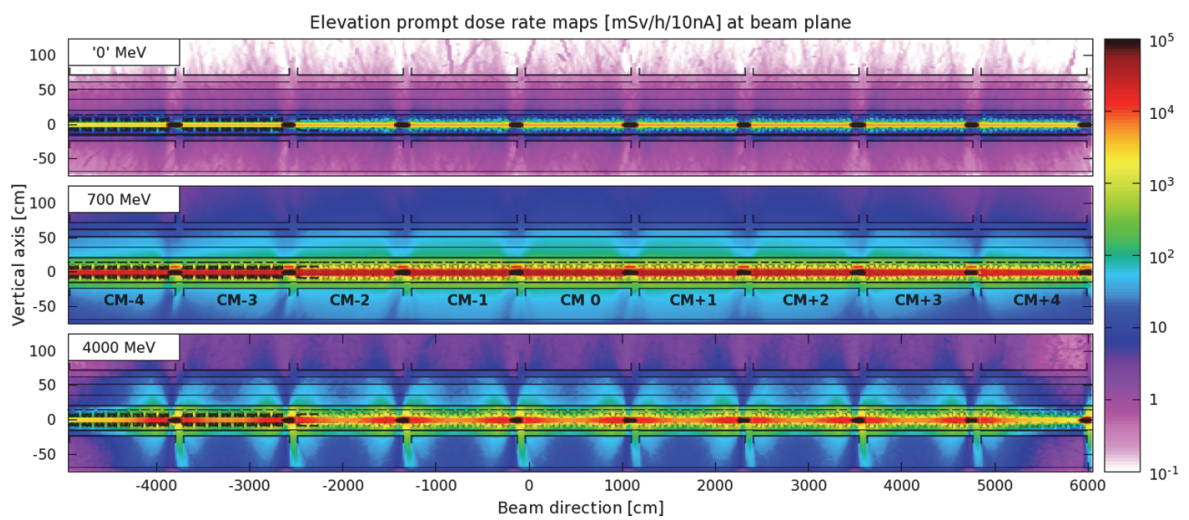

Fig. 3. Prompt dose rate (equivalent) $[\mathrm{mSv} / \mathrm{h} / 10 \mathrm{nA}]$ maps for a string of nine cryomodules with quadrupoles set at constant gradients equivalent to ' $0 \mathrm{MeV} / \mathrm{OFF}$ ', $700 \mathrm{MeV}$ and $4000 \mathrm{MeV}$.

\subsubsection{Residual dose and activation}

Dark current can simultaneously damage and radioactivate components, leading to residual radiation levels during access to the tunnel. Doses associated to maintenance tasks can be estimated by consulting the residual dose rate map of Fig. 4 (left), computed for a long irradiation cycle (10 years) and one hour cool-down, where it is observed that at the aisle, radiation levels are below the access limit of $50 \mu \mathrm{Sv} / \mathrm{h} / 10 \mathrm{nA}$.
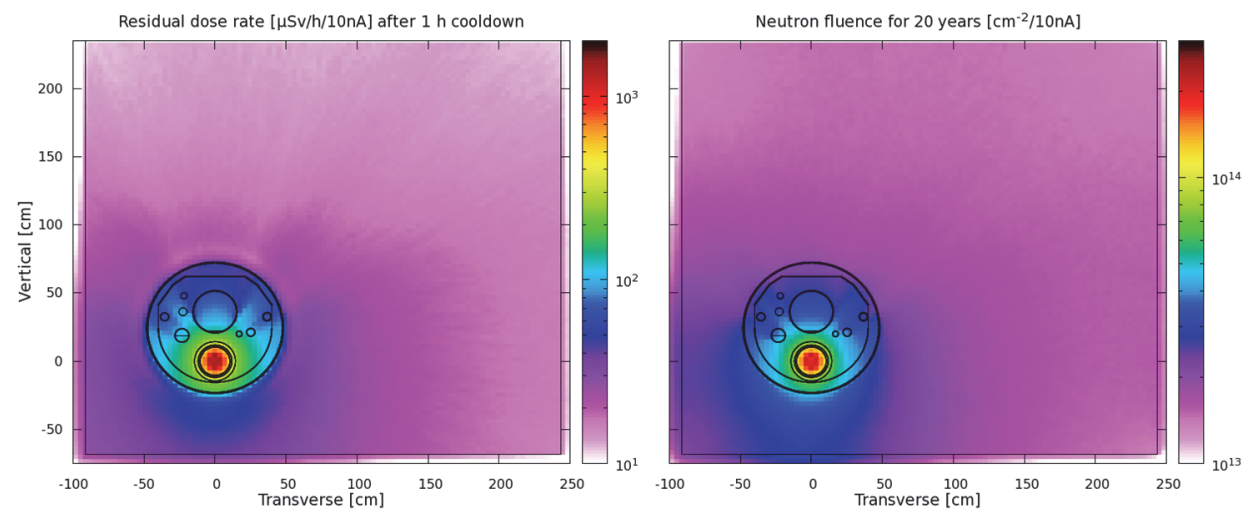

Fig. 4. Cross section maps at L2 for (left): residual dose rate $[\mu \mathrm{Sv} / \mathrm{h} / 10 \mathrm{nA}]$ after one hour of cool-down, and (right): neutron fluence over 20 year of operation $\left[\mathrm{n} / \mathrm{cm}^{2} / 10 \mathrm{nA}\right]$.

Another consideration linked with activation is the leakage of $\mathrm{He}$, which will contain tritium atoms generated through the ${ }^{4} \mathrm{He}(\gamma, p)^{3} \mathrm{H}$ reaction. Simulations were performed to compute tritium production in all He volumes (at their respective densities), leading to an average production of $8.74 \times 10^{5} \mathrm{at} / \mathrm{s} / \mathrm{nA} /$ cryomodule. Assuming a mean residence time of 
5 years, the total annual discharge of tritium will range between 1.6 and $3.4 \mathrm{MBq} / \mathrm{nA}$. This low value will be added to the National Emission Standards for Hazardous Air Pollutants (NESHAP) reports issued annually by SLAC.

\subsubsection{Dose to components in the cryomodules and in the tunnel}

Simulations assuming 300 days per year of beam operation with $10 \mathrm{nA}$ continuous dark current per cryomodule predict maximum 20-year dose levels on light plastics and silicon of 2-4 MGy inside the cryostat, 0.2-1 MGy underneath it, 0.04-0.1 MGy by the south wall, and $2-4 \mathrm{kGy}$ by the tunnel ceiling. Comparison of these values to published data, ${ }^{12}$ suggest that Teflon should not be used, and electronic components should be shielded if installed below the cryomodules. Another measure of potential radiation damage is the neutron fluence, mapped in Fig. 4 (right). According to simulations, the integrated fluence over 20 years operation will not exceed $3 \times 10^{14} \mathrm{n} / \mathrm{cm}^{2} / 10 \mathrm{nA}$, well under the threshold for most materials, and also on the safe side for specific PZT actuators used in the cryomodules, as measured in Ref. 13.

\subsubsection{Dose to quadrupoles and to the Niobium walls of the cavities}

The quadrupole magnets are wrapped around the beam pipe and deflect part of the dark current, thus receiving substantial radiation. Figure 5 (right) displays the simulated annual absorbed dose on the quadrupole cross-section, where the surface of the coils is exposed to $\sim 0.6 \mathrm{MGy}$ during 20 years operation with $10 \mathrm{nA}$ of captured currents. This estimate is below the measured threshold for Kapton ${ }^{\circledR}$ (present in the coils) degradation, which is between 1-10 MGy.
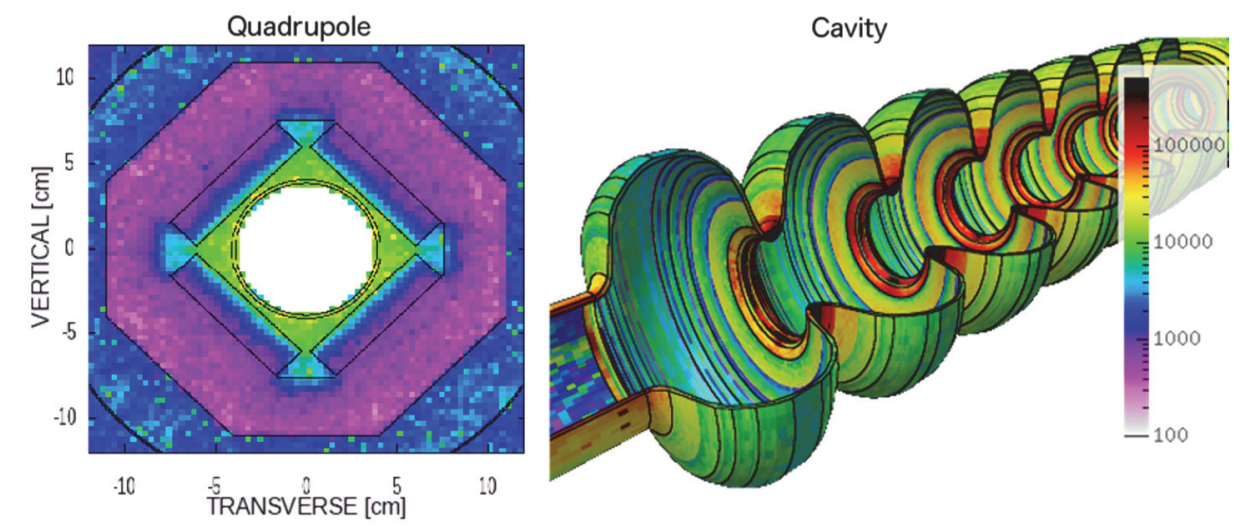

Fig. 5. Annual absorbed dose $[\mathrm{Gy} / \mathrm{nA}]$ in the quadrupole (left) or in the cavities (right) of the L2-L3 cryomodules.

The other components more prone for irradiation due to their position are the cavities. Simulations show (left image in Fig. 5) that the most irradiated areas of the cavities are the $3^{\text {rd }}$ to $5^{\text {th }}$ irises, which absorb less than $50 \mathrm{kGy} / \mathrm{nA}$ annually, thus $10 \mathrm{MGy}$ in a 20-year lifetime of the machine with $10 \mathrm{nA}$ currents. This dose is well below metal radiation 
damage thresholds (>1000 MGy). Also, the corresponding absorbed power from field emission $(\sim 15 \mathrm{~mW} / \mathrm{nA})$ is negligible compared to RF heating $(\sim 100 \mathrm{~W})$.

\section{Conclusions}

Simulations of dark current emission, acceleration, and interaction with cryomodule components show that L2 will be the most radiated section of LCLS-II. Captured current should be kept below $10 \mathrm{nA}$ /cryomodule, as otherwise, residual radiation may be too high to allow access with short cool-down times, and quadrupole coils and piezoactuators may get damaged during the facility lifetime. If quads are all off, significant dark current power could be absorbed in the upstream and downstream warm sections.

\section{Acknowledgments}

This work was supported by U.S. Department of Energy contract DE-AC02-76SF00515.

\section{References}

1. N. Dhanaraj, C. Ginsburg, I. Rakhno, G. Wu, Radiation Shielding for Superconducting RF Cavity Test Facility at A0*, Fermilab-TM-2419-APC-TD.

2. N. Akasaka, Dark Current Simulation in High Gradient Accelerating Structure, KEP Preprint 96-65, July 1996.

3. V. Balandin et al., Studies of Electromagnetic Cascade Showers Development in the TESLA Main Linac Initiated by Electron Field Emission in RF Cavities, TESLA Report 2003-10.

4. K. Ko et al., SciDAC and the International Linear Collider, Petascale Computing for Terascale Acelerator, in Proc. SciDAC 2006 Conf. (Denver, Colorado, 2006).

5. R. H. Fowler and L. Nordheim, Electron Emission in Intense Electric Fields, in Proc. Royal Society of London Series A, Vol. 119, No. 781 (1928), pp. 173-181.

6. L.Q. Lee et al., Enabling Technologies for Petascale Electromagnetic Simulations, in Proc. SciDAC 2007 (Boston, Massachusetts, 2007).

7. T.T. Böhlen, F. Cerutti, M.P.W. Chin, A. Fassò, A. Ferrari, P.G. Ortega, A. Mairani, P.R. Sala, G. Smirnov and V. Vlachoudis, The FLUKA Code: Developments and Challenges for HighEnergy and Medical Applications, Nuclear Data Sheets 120, 211-214 (2014).

8. A. Ferrari, P.R. Sala, A. Fassò, and J. Ranft, FLUKA: a multi-particle transport code, CERN2005-10 (2005), INFN/TC_05/11, SLAC-R-773.

9. V. Vlachoudis, FLAIR: A Powerful But User Friendly Graphical Interface For FLUKA, in Proc. Int. Conf. on Mathematics, Computational Methods \& Reactor Physics (M\&C 2009), (Saratoga Springs, New York, 2009).

10. H. Weise, Status of and Results from TTF Linac Operation and Outlook TTF2.

11. P. Kneisel, Cavity Preparation Techniques and Impact on Q Realistic Q-factors in a Module, Review of Modules, ERL 2005, Jefferson Lab.

12. C.L. Hanks and D.J. Hamman, Radiation Effects Design Handbook, Section 3. Electrical Insulating Materials and Capacitors, NASA-CR-1787.

13. M. Fouaidy et al., Electromechanical, Thermal Properties and Radiation Hardness tests of Piezoelectric Actuators at low Temperature, in Proc. $12^{\text {th }}$ Int. Workshop on $R F$ Superconductivity (Cornell University, 2005). 